\title{
Multimode interference in tapered single mode-multimode-single mode fiber structures for strain sensing applications
}

\author{
Ricardo M. André*a, ${ }^{a,}$ Claudecir R. Biazoli ${ }^{\mathrm{c}}$, Susana O. Silva ${ }^{\mathrm{a}, \mathrm{b}}$, Manuel B. Marques ${ }^{\mathrm{a}, \mathrm{b}}$, \\ Cristiano M. B. Cordeiro ${ }^{c}$, Orlando Frazão ${ }^{a}$

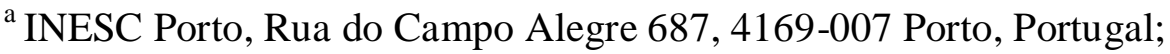 \\ ${ }^{\mathrm{b}}$ Departamento de Física da Faculdade de Ciências da Universidade do Porto, Rua do Campo Alegre \\ 687, 4169-007 Porto, Portugal \\ c Instituto de Física “Gleb Wataghin”, Universidade Estadual de Campinas - UNICAMP, Campinas, \\ SP, Brazil
}

\begin{abstract}
Tapering single mode-multimode-single mode structures to enhance sensitivity is proposed and experimentally demonstrated. $50 \mathrm{~mm}$-long coreless MMF sections are spliced between SMFs and tapered. They are characterized in strain and an increase in strain sensitivity is obtained with taper diameter reduction. Sensitivities as high as $-23.69 \mathrm{pm} / \mu \varepsilon$ for the $15 \mu \mathrm{m}$ taper are attained. A combination of an untapered and tapered SMS is proposed as a sensing system for the simultaneous measurement of strain and temperature.
\end{abstract}

Keywords: fiber optics sensors, multimode interference.

\section{INTRODUCTION}

Multimode interference (MMI) has been thoroughly investigated and many applications namely in integrated optics have sprouted from such concept. Self-imaging of the input light field has led to the development of directional couplers, beam splitters, and multiplexers just to name a few [1]. Multimode interference can also be obtained in optical fibers using a step-index multimode fiber (MMF) and can be used for optical communication and sensing [2]. The most usual form of an MMI-based optical fiber device consists of a multimode fiber section spliced between two single-mode fibers (SMF) forming an SMF-MMF-SMF structure (SMS) [3]. SMS fiber structures have been employed as many optical devices such as a displacement sensor [4], a fiber lens [5], refractometer sensors [6], bandpass filters [7], and edge filters [8]. Recently, Wang et al. proposed and experimentally demonstrated a high sensitivity evanescent field fiber refractometer based on a tapered multimode-single-mode fiber structure [9].

This work presents a tapered SMS structure that acts as a multimode interference device. The sensing head is composed by a $50 \mathrm{~mm}$-long section of coreless multimode fiber, spliced between to SMF 28. On the coreless multimode section, tapers with different cross sections ranging from the untapered $125 \mu \mathrm{m}$ to $15 \mu \mathrm{m}$ were fabricated. Simulations of a conventional SMS structure and one that includes a taper in the multimode region were compared. Different SMS sensing heads with different taper dimensions were characterized in strain.

\section{SETUP AND SIMULATIONS}

The sensing heads are composed of a piece of coreless multimode silica fiber spliced between two single-mode optical fibers (see Figure 1). On the coreless multimode fiber, a taper is produced by the flame-brushing technique. This technique employs a flame that is swept along the fiber while it is being stretched and thus leads to the reduction of the transverse cross-section and the creation of a taper. 


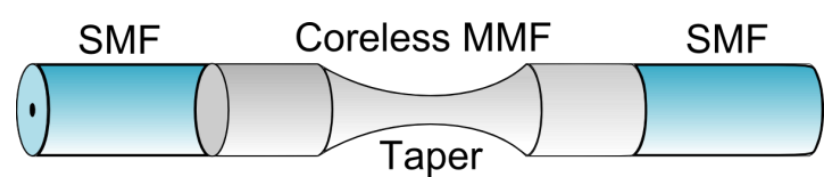

Figure 1. SMS structure with a taper in the multimode region.

The structure will act as a multimode interference device (MMI). In Figure 2, simulations of the untapered structure (a) and of the structure tapered down to $55 \mu \mathrm{m}$ (b) are presented. The simulation was performed using a Beam Propagation Method (BPM). The parameters used in the simulation are light injected at $1550 \mathrm{~nm}, 50 \mathrm{~mm}-$ long section of $125 \mu \mathrm{m}$ coreless fiber with a refractive index of 1.444. In Figure 2 (a) one can see the usual MMI pattern along the $50 \mathrm{~mm}$ of coreless fiber. In Figure 2 (b), it is evident that even though the taper changes the propagation of light inside the fiber, the MMI pattern is still clearly visible.

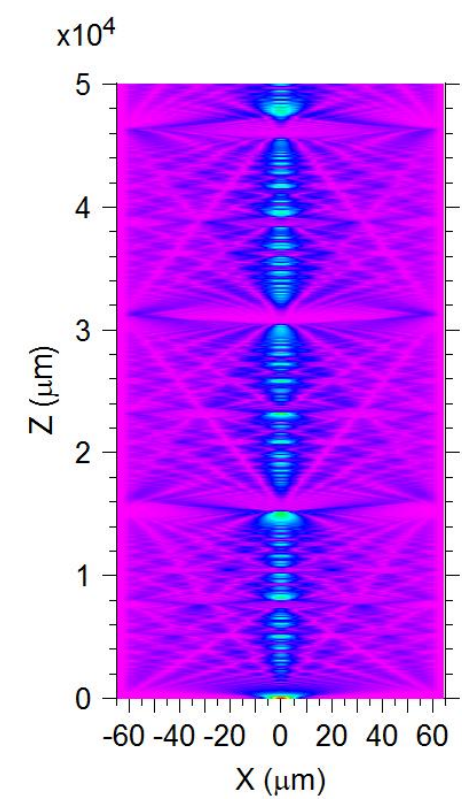

(a)

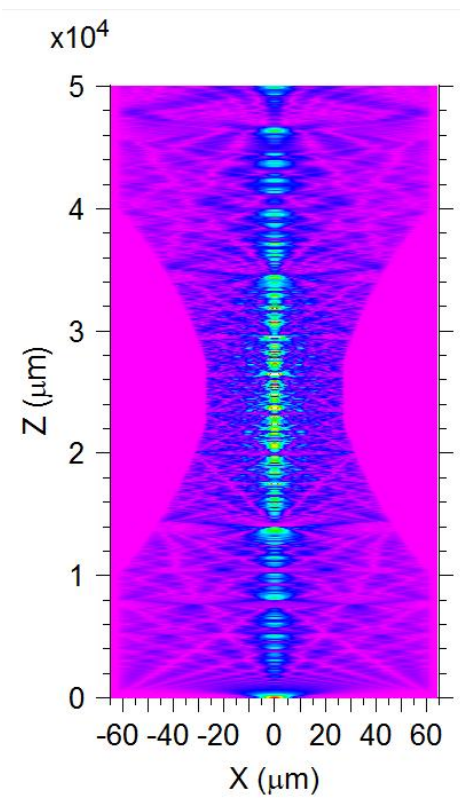

(b)

Figure 2. BPM calculated electric field amplitude distribution in the $X Z$ plane for (a) untapered and (b) tapered coreless MMF sandwiched between two standard SMFs. The length of the untapered MMF is $50 \mathrm{~mm}$; the diameter and length of the uniform waist are $55 \mu \mathrm{m}$ and $5 \mathrm{~mm}$ respectively; and the operating wavelength is $1550 \mathrm{~nm}$.

\section{EXPERIMENTAL RESULTS}

When analyzing the spectral responses of the several SMS structure with different taper waists, one can identify, for small diameter reduction (large taper waists), a clear MMI image in the spectrum (see Figure 3 (a) and (b)). As the taper waist is reduced a smaller number of propagating modes is expected. This is seen in Figure 3 (b) because a larger bandwidth is visible which results from a smaller number of modes. When going to smaller taper waists $(25 \mu \mathrm{m}$ and 15 $\mu \mathrm{m})$, the number of modes that propagate through the taper region should be even smaller but the intermodal interference present does not allow us to take such conclusions (Figure 3(c)).

For strain characterization, each SMS structure was placed on micrometric translation stages and subjected to controlled stretching. The total length of the sensing head $(0.4 \mathrm{~m})$ was subjected to strain. To determine the wavelength variations, the wavelength change of the main peaks was monitored through an Optical Spectrum Analyzer with a maximum resolution of $0.05 \mathrm{~nm}$. 


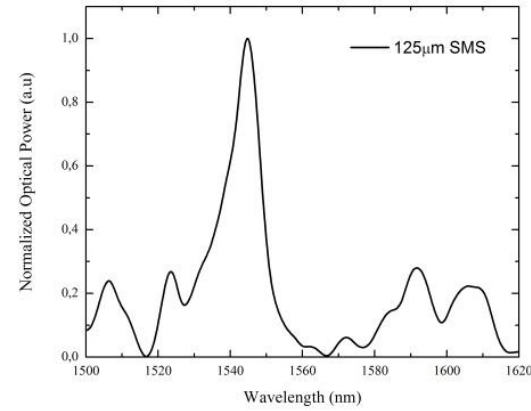

(a)

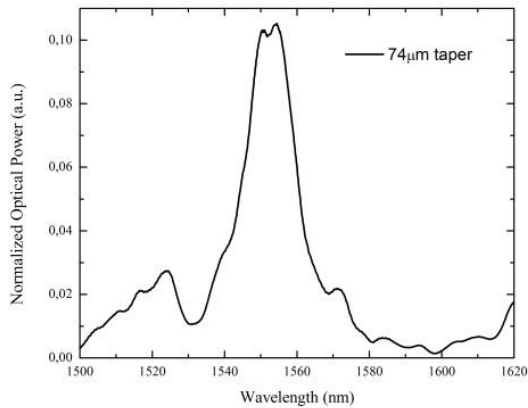

(b)

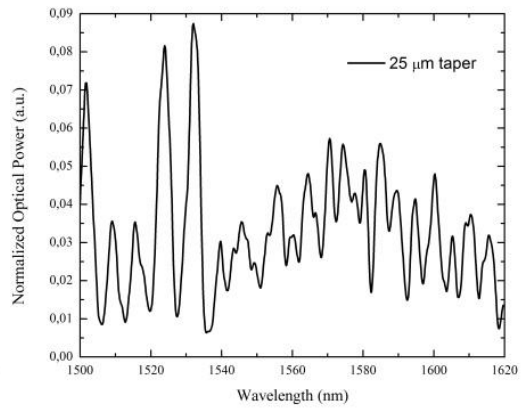

(c)

Figure 3. Spectral responses of the $125 \mu \mathrm{m}$ SMS (a), and the tapered $74 \mu \mathrm{m}$ (a) and $25 \mu \mathrm{m}$ (b) SMSs.

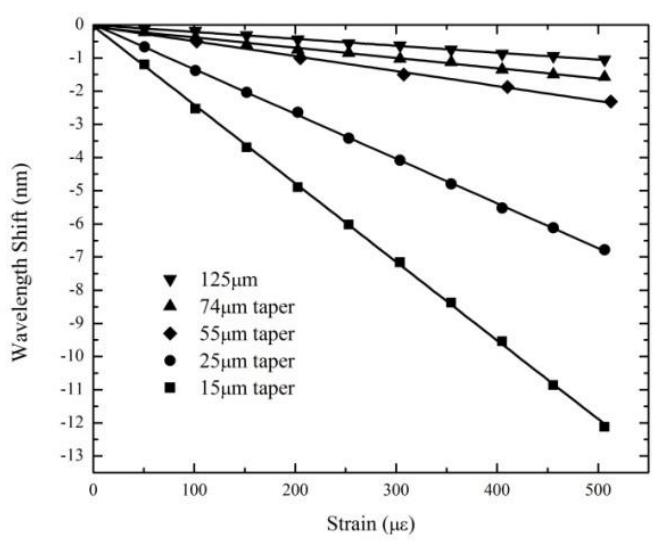

(a)

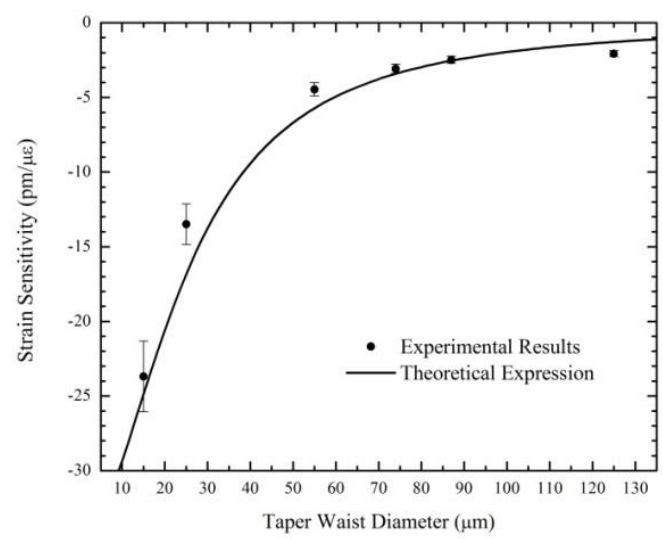

(b)

Figure 4. (a) Wavelength shift vs. applied strain for the several taper structures; (b) Relationship between strain sensitivity and taper size.

The theoretical expression plotted in Figure 4 (b) and given by equation (1) is derived from a purely geometrical point of view and is a mere estimate of the strain sensitivity of such structure namely FBGs and LPGs [10, 11]. Strain sensitivity varies nonlinearly with taper waist diameter. It increases as taper diameter decreases: from $-2.08 \mathrm{pm} / \mu \varepsilon$ for the untapered SMS to $-23.69 \mathrm{pm} / \mu \varepsilon$ for the $15 \mu \mathrm{m}$ tapered SMS.

$$
\kappa=\kappa_{\varepsilon \text { (taper })} \frac{L_{\text {taper }}+L_{\text {fiber }}}{L_{\text {fiber }} \frac{d_{\text {taper }}^{2}}{d_{\text {fiber }}^{2}}+L_{\text {taper }}}
$$

These tapered SMS structures can be used for the simultaneous measurement of temperature and strain using two different diameter tapers. A solution is presented using the matrix method. It should be taken into account that the material is always the same, thus it is expected that the temperature sensitivity will also be approximately the same and independent of the taper diameter $\left(\kappa_{T(125)} \approx \kappa_{T(15)} \approx \kappa_{T}\right.$ ). Considering, for example, the $125 \mu \mathrm{m}$ and the $15 \mu \mathrm{m}$ tapers one has:

$$
\left[\begin{array}{c}
\Delta \lambda_{S M S(125)} \\
\Delta \lambda_{S M S(15)}
\end{array}\right]=\left[\begin{array}{ll}
\kappa_{T(125)} & \kappa_{\varepsilon(125)} \\
\kappa_{T(15)} & \kappa_{\varepsilon(125)}
\end{array}\right]\left[\begin{array}{c}
\Delta T \\
\Delta \varepsilon
\end{array}\right]
$$

Inverting the matrix one can obtain expressions for the temperature and strain variations as a function of the sensitivities and wavelength shifts: 


$$
\Delta T=\frac{\kappa_{\varepsilon(15)} \Delta \lambda_{(125)}-\kappa_{\varepsilon(125)} \Delta \lambda_{(15)}}{\kappa_{T}\left(\kappa_{\varepsilon(15)}-\kappa_{\varepsilon(125)}\right)} ; \quad \Delta \varepsilon=\frac{\Delta \lambda_{(15)}-\Delta \lambda_{(125)}}{\kappa_{\varepsilon(15)}-\kappa_{\varepsilon(125)}}
$$

\section{CONCLUSIONS}

Tapers were produced on SMS structures and were characterized in strain. From the simulations it was ascertained that the creation of a taper in the MMF section of the SMS does not destroy the MMI pattern. In fact, what happens is that the self-images appear more closely packed in the taper region. An increase of $1039 \%$ in strain sensitivity was achieved when tapering the SMS structure from $125 \mu \mathrm{m}(-2.08 \mathrm{pm} / \mu \varepsilon)$ to $15 \mu \mathrm{m}(-23.69 \mathrm{pm} / \mu \varepsilon)$. Combining two SMS structures, for example, one untapered and one tapered, it is possible to measure strain and temperature simultaneously.

\section{ACKNOWLEDGMENTS}

This work was partially supported by a bi-national cooperation project, CAPES-FCT Brazil/Portugal (\# 293/11, coordinated by authors C.M.B.C.). The authors of this paper would also like to thank Fundação Calouste Gulbenkian for the research grant awarded to Ricardo Melo André under the award "Prémio de Estímulo à Investigação". This work is also support by funding FEDER through Programa Operacional Factores de Competitividade - COMPETE e por Fundos Nacionais através da FCT - Fundação para a Ciência e a Tecnologia by the project: PTDC/EMEPME/102806/2008.

\section{REFERENCES}

[1] L. Soldano and E. Pennings, "Optical multi-mode interference devices based on self-imaging: principles and applications," Lightwave Technology, Journal of 13, pp. 615 -627, April 1995.

[2] A. Kumar, R. K. Varshney, S. A. C, and P. Sharma, "Transmission characteristics of sms fiber optic sensor structures," Optics Communications 219, pp. 215 -219, 2003.

[3] Q. Wang, G. Farrell, and W. Yan, "Investigation on single-mode-multimode-single-mode fiber structure," $J$. Lightwave Technol. 26, pp. 512-519, Mar 2008.

[4] A. Mehta, W. Mohammed, and E. Johnson, "Multimode interference-based fiber-optic displacement sensor," Photonics Technology Letters, IEEE 15, pp. 1129-1131, aug. 2003.

[5] W. Mohammed, A. Mehta, and E. Johnson, "Wavelength tunable fiber lens based on multimode interference," Lightwave Technology, Journal of 22, pp. 469 - 477, feb. 2004.

[6] O. Frazão, S. O. Silva, J. Viegas, L. A. Ferreira, F. M. Araújo, and J. L. Santos, "Optical fiber refractometry based on multimode interference," Appl. Opt. 50, pp. E184-E188, Sep 2011.

[7] W. S. Mohammed, P. W. E. Smith, and X. Gu, "All-fiber multimode interference bandpass filter," Opt. Lett. 31, pp. 2547-2549, Sep 2006.

[8] Q. Wang and G. Farrell, "Multimode-fiber-based edge filter for optical wavelength measurement application and its design," Microwave and Optical Technology Letters 48(5), pp. 900-902, 2006.

[9] P. Wang, G. Brambilla, M. Ding, Y. Semenova, Q. Wu, and G. Farrell, "High-sensitivity, evanescent field refractometric sensor based on a tapered, multimode fiber interference," Opt. Lett. 36, pp. 2233-2235, Jun 2011.

[10] O. Frazão, S. F. O. Silva, A. Guerreiro, J. L. Santos, L. A. Ferreira, and F. M. Araújo, "Strain sensitivity control of fiber bragg grating structures with fused tapers," Appl. Opt. 46(36), pp. 8578-8582, 2007.

[11] C. R. Biazoli, R. M. André, O. Frazão, C. M. B. Cordeiro, "Tapering down optical fiber to ultra-high strain sensitivity", Photonics Technology Letters, IEEE , 2012. (Submitted). 